\title{
AVALIAÇÃO PROSPECTIVA DOS RESULTADOS A LONGO PRAZO DA TERAPIA INTRADISCAL PERCUTÂNEA LOMBAR POR RADIOFREQUÊNCIA
}

\author{
PROSPECTIVE EVALUATION OF LONG-TERM RESULTS OF PERCUTANEOUS LUMBAR \\ INTRADISCAL RADIOFREQUENCY THERAPY
}

\author{
EVALUACIÓN PROSPECTIVA DE RESULTADOS A LARGO PLAZO DE LA TERAPIA \\ INTRADISCAL PERCUTÁNEA LUMBAR CON RADIOFRECUENCIA
}

Daniel Ferreira Ghedini¹, Rogério Lúcio Chaves de Resende ${ }^{1}$, Túlio Vinícius de Oliveira Campos², Jefferson Soares Leal ${ }^{3}$, Otávio de Lucca Druda ${ }^{4}$, Marco Antônio Percope de Andrade ${ }^{5}$

\begin{abstract}
RESUMO
Objetivo: Avaliar os resultados do tratamento da lombalgia crônica discogênica com a técnica IDET (Intradiscal Electrothermal Therapy) em um acompanhamento maior que dois anos de pós-operatório (PO). Métodos: Estudo prospectivo, avaliando 23 pacientes consecutivos com diagnóstico de lombalgia crônica discogênica tratados com IDET entre fevereiro de 2003 e fevereiro de 2006. A dor foi considerada discogênica em caso de discografia positiva ou imagem compatível com a ressonância magnética, segundo os critérios de Horton e Daftari. Os parâmetros clínicos (EVA e Oswestry) foram avaliados no pré-operatório, no retorno com 6, $12,18,24$ e mais de 48 meses de pós-operatório. Resultados: A média de idade dos pacientes foi de 42,09 (variação de 26 a 51 anos). A média do período do acompanhamento foi de 32,55 meses (variação de 6 a 68 meses). A média do índice de dor (EVA) foi de 7,07 $\pm 1,90$, reduzindo-se para 2,47 $\pm 1,876$ em 6 meses, 2,69 $\pm 1,82$ em 12 meses, 3,63 $\pm 1,75$ em 18 meses e 5,73 $\pm 2,12$ em 24 meses. A média do índice Oswestry

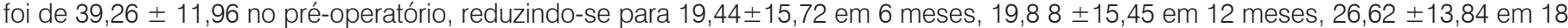
meses e 31,87 $\pm 14,94$ em 24 meses. Os resultados não se mantiveram no acompanhamento de 48 meses. Conclusão: 0 IDET mostrou resultados satisfatórios como alternativa paliativa no controle da dor e da incapacidade funcional num período de 24 meses. Os bons resultados deterioraram-se com 48 meses e houve uma tendência de retorno à sintomatologia inicial.
\end{abstract}

Descritores: Dor lombar; Imagem por ressonância magnética; Medição da dor. Estudos prospectivos; ablação por cateter.

\begin{abstract}
Objectives: Evaluate the results of treatment of patients with chronic discogenic low back pain who have been submitted to IDET (Intradiscal Electrothermal Therapy). Minimum post operatory follow up was two years. Methods: Prospective study of 23 patients diagnosed with chronic discogenic low back pain who received treatment between February 2003 and February 2006. The disk was considered the source of the pain in case of a positive discography or a nuclear magnetic resonance image compatible with Horton and Daftari criteria. Clinical parameters (VAS and Oswestry) were evaluated during pre-operatory and at 6, 12, 18, 24 and 48 months after surgery. Results: The average age of patients was 42,09 years (26 to 51 years). The mean follow up time was 32,55 months (6 to 68 months). The mean visual analog scale (VAS) was 7,07 $\pm 1,90$, and was reduced to 2,47 $\pm 1,876$ at 6 months, 2,69 $\pm 1,82$ at 12 months, 3,63 $\pm 1,75$ at 18 months and 5,73 $\pm 2,12$ at 24

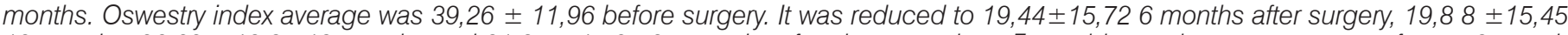
12 months, 26,62 $\pm 13,8418$ months and 31,87 $\pm 14,9424$ months after the procedure. Favorable results were not seen after a 48-month follow up. Conclusion: IDET technique has proved to be a good alternative in the palliative control of pain and functional incapacity during a 24-month period. Good results have deteriorated after 48 months, with a tendency to return of symptoms as they were prior to treatment.
\end{abstract}

Keywords: Low back pain; Magnetic resonance imaging; Pain measurement; Prospective studies; Catheter ablation.

\section{RESUMEN}

Objetivo: Evaluar los resultados del tratamiento del lumbago crónico del disco usando la técnica IDET (electrotermocoagulación intradiscal) en un seguimiento mayor que dos años de pos operatorio. Método: Estudio prospectivo, evaluando 23 pacientes, consecutivamente, con diagnóstico de lumbago crónico del disco tratados con IDET desde febrero de 2003 hasta febrero de 2006. El disco fue considerado como siendo la fuente del dolor en caso de discografia positiva o imagen compatible con la resonancia magnética según los criterios de Horton y Daftari. Los parámetros clínicos (EVA y Oswestry) fueran evaluados en el pre operatorio, en el retorno, con 6, 12, 18, 24 y más de 48 meses de pos operatorio. Resultados: El promedio de edad de los pacientes fue 42.09 años (variación de 26 a 51 años). El promedio del período del seguimiento fue 32,55 meses (variación de 6 a 68 meses). El promedio del Índice de Dolor (EVA) fue de 7,07 $\pm 1,90$ reduciéndose para 2,47 $\pm 1,876$ en 6 meses, 2,69 1,82 en 12 meses, 3,63 $\pm 1,75$ en 18 meses y 5,73 $\pm 2,12$ en 24 meses. El promedio del Índice Oswestry fue de $39,26 \pm 11,96$

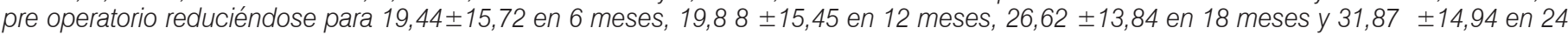
meses. Conclusión: La IDET mostró resultados satisfactorios como alternativa paliativa para el control del dolor y de la incapacidad funcional en un periodo de 24 meses. Los buenos resultados se deterioraron con 48 meses y hubo una tendencia de retorno de los síntomas iniciales.

Descriptores: Dolor en la región lumbar; Imagen por resonancia magnética Medición del dolor; Estudios prospectivos; Ablación por catéter.

1 - Médico assistente do Grupo de Coluna do Biocor Instituto, Hospital das Clínicas da Universidade Federal de Minas Gerais, Belo Horizonte, MG, Brasil.

2 - Médico assistente do Biocor, Hospital das Clínicas da Universidade Federal de Minas Gerais, Belo Horizonte, MG, Brasil.

3 - Mestre; Coordenador do Grupo de Coluna do Serviço de Ortopedia do Biocor, Hospital das Clínicas da Universidade Federal de Minas Gerais, Belo Horizonte, MG, Brasil.

4 - Especializando em Cirurgia da Coluna do Biocor, Hospital das Clínicas da Universidade Federal de Minas Gerais, Belo Horizonte, MG, Brasil.

5 - Doutor; Chefe do Serviço de Ortopedia eTraumatologia do Biocor, Hospital das Clínicas da Universidade Federal de Minas Gerais, Belo Horizonte, MG, Brasil.

Trabalho realizado no Serviço de Ortopedia do Hospital das Clínicas da Universidade Federal de Minas Gerais e Biocor Instituto, MG, Brasil.

Correspondência: Av. do Contorno, 5057, Belo Horizonte, MG. Brasil. CEP 30110-100. E-mail: ghedinidg@yahoo.com 


\section{INTRODUÇÃO}

A lombalgia pode ser definida como uma sensação desagradável ou desconforto na região inferior do dorso que eventualmente se estende para as nádegas ${ }^{1}$. Em países industrializados é a segunda doença mais frequente e invariavelmente leva a absenteísmo no trabalho ${ }^{2-4}$.

Cerca de 60 a 80\% da população experimentará pelo menos um episódio de lombalgia durante a vida ${ }^{5}$. $90 \%$ dos pacientes melhoram em três meses, entretanto 5\% evoluem para lombalgia crônica e $60 \%$ têm um quadro recorrente ${ }^{6}$.

A definição da causa anatômica da lombalgia é difícil, todavia trabalhos experimentais mostram que em até $40 \%$ dos casos essa é encontrada no disco intervertebral ${ }^{7-9}$. A literatura não consegue definir correlação precisa entre as alterações degenerativas discais e a dor lombar ${ }^{10-13}$.

O termo "lesão discal interna", descrito por Crock $^{14,15}$, é usado para identificar uma síndrome caracterizada por lombalgia, sem sintomas radiculares, causada por doença discal degenerativa e sem manifestação radiológica. O tratamento da lombalgia permanece como um desafio ${ }^{16,17}$. Tradicionalmente, o tratamento da dor lombar crônica causada por doença discal tem sido limitado à fisioterapia, uso de medicamentos analgésicos além de métodos alternativos sem fundamentação científica. Nos casos de dor incapacitante, a artrodese do segmento envolvido tem sido a cirurgia tradicionalmente indicada ${ }^{18,19}$. Os resultados da artrodese são muito variáveis ${ }^{17-19}$ e estudos randomizados mostram que a fusão não é muito mais eficaz do que a terapia não invasiva ${ }^{20}$.

O IDET (Intradiscal Electrothermal Therapy) proposto em 1997 baseia-se na hipótese de que a energia térmica poderia tratar a lesão discal interna (LDI) e a dor por desnaturar o do colágeno presente no disco, cauterizar o tecido de granulação e coagular as terminações nervosas neoformadas ${ }^{17}$.
O propósito deste estudo é avaliar prospectivamente os resultados a longo prazo do IDET em uma série de casos de lombalgia crônica discogênica sem resposta ao tratamento conservador.

\section{MATERIAIS E MÉTODOS}

Vinte três pacientes foram submetidos ao IDET e avaliados prospectivamente no $6^{\circ}, 12^{\circ}, 18^{\circ}, 24^{\circ}$ e $48^{\circ}$ meses de pós-operatório (Tabela 1). Foram incluídos no estudo pacientes com lombalgia crônica discogênica com duração superior a seis meses e refratária ao tratamento conservador. A origem da dor foi confirmada por discografia ou segundo critérios de Horton e Daftari ${ }^{21}$. Foram excluídos pacientes com radiculopatia, estenose lombar, deformidade espinhal, cirurgia prévia na coluna lombar, instabilidade do segmento vertebral, doenças inflamatórias sistêmicas e gestantes. Todos os procedimentos foram realizados entre fevereiro de 2003 e fevereiro de 2006 pelo mesmo cirurgião (JSL).

Todos pacientes foram submetidos ao exame de ressonância nuclear magnética (RNM) da coluna lombar, avaliação pré-operatória da intensidade da dor por escala visual analógica (EVA - régua ilustrada e com escala de 0 a 10 no seu verso) e do grau de incapacidade funcional pelo índice de Oswestry²2.

As variáveis clínicas registradas foram: sexo, idade, discografia, existência de litígio trabalhista, nível(is) discal(is) abordado(s) e satisfação do paciente. A obtenção da resposta era feita através de questionário autoaplicado no retorno.

Sete pacientes foram submetidos à discografia pela técnica padrão preconizada pela International Spinal Injection Society ${ }^{23}$. A discografia foi considerada positiva se o paciente apresentava dor concordante com sua queixa quando $2,5 \mathrm{ml}$ de contraste (lopamiron $^{\circledR}$ - Bayer) foi injetado no disco sob suspeita e com nível de controle negativo (disco adjacente normal). Nos demais pacientes $(n=16)$ a identificação do nível causador da dor foi realizada pela presença de sinais indicativos na RNM segundo Horton e Daftari²1.

Tabela 1. Casos submetidos ao tratamento com IDET.

\begin{tabular}{|c|c|c|c|c|c|c|c|c|c|c|c|c|c|c|c|}
\hline \multirow[b]{3}{*}{ ষ্ণ } & \multirow[b]{3}{*}{ 㟯 } & \multirow[b]{3}{*}{ 趉 } & \multirow[b]{3}{*}{ 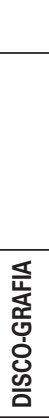 } & \multicolumn{2}{|c|}{ PRÉ-OPERATÓRIO } & \multicolumn{10}{|c|}{ PÓS-OPERATÓRIO } \\
\hline & & & & & & \multicolumn{2}{|c|}{ 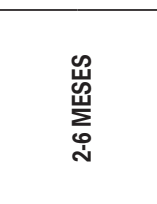 } & \multicolumn{2}{|c|}{ 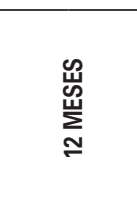 } & \multicolumn{2}{|c|}{ 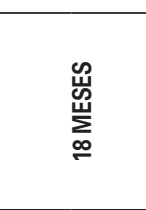 } & \multicolumn{2}{|c|}{ 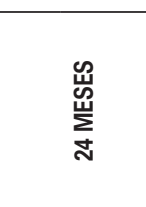 } & \multicolumn{2}{|c|}{ 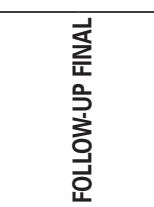 } \\
\hline & & & & 䍃 & 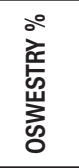 & 焉 & 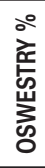 & 舀 & 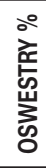 & 営 & 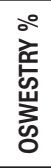 & 点 & 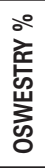 & 獣 & 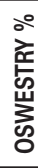 \\
\hline 1 - MJP & 36 & M & $\mathrm{N}$ & 6 & 22 & 0 & 0 & 0 & 0 & 2 & 10 & 5 & 20 & 10 & 64 \\
\hline 2 - ISF & 45 & M & N & 5 & 50 & 4 & 36 & 4 & 34 & 5 & 38 & 5 & 36 & 8 & 60 \\
\hline $3-I F N$ & 36 & $\mathrm{~F}$ & N & 5 & 40 & 1 & 10 & 3 & 14 & 5 & 16 & 6 & 36 & - & - \\
\hline 4-FMBS & 33 & M & N & 8,5 & 52 & 3 & 18 & 3 & 18 & 3 & 16 & 6 & 20 & - & 60 \\
\hline 5 - EMSC & 26 & $\mathrm{~F}$ & $\mathrm{~N}$ & 5 & 40 & - & - & - & - & 3 & - & - & - & - & - \\
\hline $6-C A G$ & 36 & M & N & 7 & 50 & 4 & 32 & 5 & 38 & - & 40 & 52 & 52 & - & - \\
\hline 7 - IDFF & 50 & M & N & 10 & 64 & 5 & 38 & 6 & 42 & 6 & 38 & 50 & 50 & - & - \\
\hline 8 -EFD & - & M & $N$ & 7 & 22 & 0 & - & - & - & 5 & - & - & - & 10 & - \\
\hline $9-\mathrm{TCA}$ & 57 & M & $N$ & 10 & - & 5 & 60 & 5 & 50 & 5 & 50 & - & - & - & 40 \\
\hline $10-$ RSS & 44 & M & S & 4 & 24 & 0 & 0 & 0 & 0 & 0 & 0 & 22 & 22 & - & 0 \\
\hline $11-$ RBO & 47 & M & S & 9 & 44 & 6 & 30 & - & - & - & - & - & - & 10 & - \\
\hline $12-\mathrm{RML}$ & 49 & M & $\mathrm{N}$ & 7 & 22 & - & - & - & - & - & - & - & - & 7 & si- \\
\hline $13-R G S$ & 35 & M & S & 4 & 48 & 1 & 10 & 2 & 10 & 2 & 10 & 18 & 18 & - & 88 \\
\hline 14 - MRLA & 46 & $\mathrm{~F}$ & $\mathrm{~N}$ & 6 & 22,5 & - & - & - & - & - & - & - & - & - & 4 \\
\hline $15-M L S$ & 51 & $\mathrm{~F}$ & $\mathrm{~N}$ & 5 & 34 & 1 & 14 & - & - & - & - & - & - & - & 44 \\
\hline $16-S G$ & 40 & M & $S$ & 9,5 & 50 & 4 & 26 & 4 & 30 & 5 & 36 & 50 & 50 & - & - \\
\hline 17 - GRS & 39 & M & $S$ & 9 & 52 & 3 & 22 & 3 & 22 & 6 & 30 & 48 & 48 & - & - \\
\hline 18 - MPS & 41 & $\mathrm{~F}$ & $S$ & 9 & 34 & 3 & 20 & 3 & 24 & 4 & 24 & 34 & 34 & - & . \\
\hline $19-\mathrm{MCPS}$ & 43 & $\mathrm{~F}$ & $\mathrm{~N}$ & 6 & 36 & 0 & 0 & 1 & 0 & 1 & 10 & 0 & 0 & - & - \\
\hline $20-J M$ & 44 & $\mathrm{~F}$ & N & 7 & 38 & 1 & 6 & 1 & 16 & 4 & 24 & 36 & 36 & - & - \\
\hline 21 - MVA & 42 & M & $S$ & 7,5 & 40 & 1 & 10 & 1 & 10 & 4 & 20 & 20 & 20 & - & - \\
\hline $22-O D M$ & 42 & $\mathrm{~F}$ & N & 7 & 40 & 2 & 18 & 2 & 10 & 3 & 16 & 36 & 36 & - & - \\
\hline $23-\mathrm{MHLS}$ & 44 & $\mathrm{~F}$ & $N$ & 9 & - & 3 & - & - & - & - & - & - & - & - & - \\
\hline
\end{tabular}


Os procedimentos foram realizados em ambiente cirúrgico sob condições assépticas, sedação leve e anestesia local. Através da radioscopia, foi posicionado o fio guia do instrumental do IDET no disco a ser tratado. A seguir, o eletrodo flexível de radiofrequência $\left(\right.$ Smith Nephew ${ }^{\circledR}$ - gerador de radiofrequência Oratec ${ }^{\circledR}$ ) foi inserido e seu posicionamento confirmado por radioscopia. A temperatura do eletrodo foi gradualmente aumentada até $90^{\circ} \mathrm{C}$ durante 13 minutos e mantida nessa temperatura por 4 minutos. Ao final do procedimento $1 \mathrm{~mL}$ Bupivacaína (Marcaína ${ }^{\circledR}$ - 5mg/mL) foi injetada no disco para analgesia. Profilaxia para infecção foi realizada com 2 gramas de Cefazolina $\left(\right.$ Kefazo $\left.^{\circledR}\right)$ intravenosa.

No pós-operatório o paciente foi liberado para realizar as tarefas do cotidiano, restringindo-se atividades esportivas de impacto e levantamento de peso por três meses.

Foi realizado uma busca ativa dos pacientes submetidos ao IDET que apresentavam follow-up maior que 48 meses. Foram localizados oito pacientes.

Quatro pacientes (16\%) foram submetidos à artrodese $360^{\circ}$. Esses pacientes tiveram a data da cirurgia como data final do follow-up. Esses pacientes operados foram considerados como falha do IDET.

A análise estatística dos dados foi realizada no programa SPSS for Windows versão 10.0. As variáveis contínuas, após teste de normalidade foram comparadas utilizando-se o teste $t$ pareado.

\section{RESULTADOS}

A média de idade da população foi de 42,09 $\pm 6,87$, sendo 10 mulheres (43,4\%) e 13 homens (57,6\%). 34,7\% dos pacientes $(n=8)$ possuíam litígio trabalhista. O follow-up médio foi de 32,55 meses (variação de 6 a 68 meses).

Os valores médios do índice Oswestry no pré-operatório foram

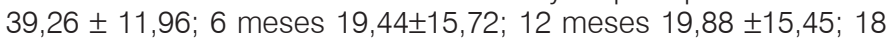
meses 26,62 $\pm 13,84 ; 24$ meses 31,87 $\pm 14,94$ e 48 meses com 45,00 $\pm 30,22$ (Figura 1). A comparação dos valores médios do Oswestry com o pré-operatório foi estatisticamente significativa com valores de $\mathrm{p}$ iguais a 0,0001 para o $6^{\circ}$ mês, 0,0004 para o $12^{\circ}$ mês, 0,002 para o $18^{\circ}$ mês e 0,032 para o $24^{\circ}$ mês. A comparação entre valor médio do Oswestry no $48^{\circ}$ mês e o pré-operatório não foi estatisticamente significativa $(p=0,098)$.

Os valores médios do índice EVA no pré-operatório foram: 7,07 $\pm 1,90 ; 6$ meses $2,47 \pm 1,87 ; 12$ meses 2,69 $\pm 1,82 ; 18$ meses 3,63 $\pm 1,75 ; 24$ meses 5,73 $\pm 2,12$ e 48 meses com 3,25 $\pm 2,25$ (Figura 2). A comparação dos valores médios da EVA com o pré-operatório foi estatisticamente significativa com valores de $p$ iguais a 0,001 para o $6^{\circ}$ mês, 0,018 para o $12^{\circ}$ mês, 0,006 para o $18^{\circ}$ mês e 0,014 para o $24^{\circ}$ mês. A comparação entre valor médio do EVA no $48^{\circ}$ mês e o pré-operatório não foi estatisticamente significativa $(p=0,882)$.

O índice de satisfação com o tratamento proposto foi de $78 \%$ (18 em 23 pacientes que responderam a esta avaliação) no $6^{\circ}$ mês

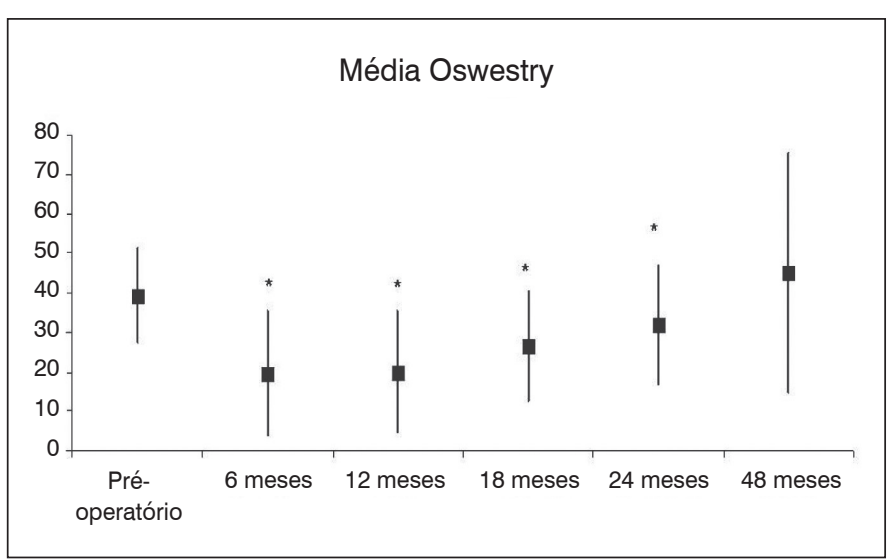

Figura 1. Valores médios do índice Oswestry ao longo do estudo, barra de erros representa o desvio padrão do grupo, * $p<0,05$.

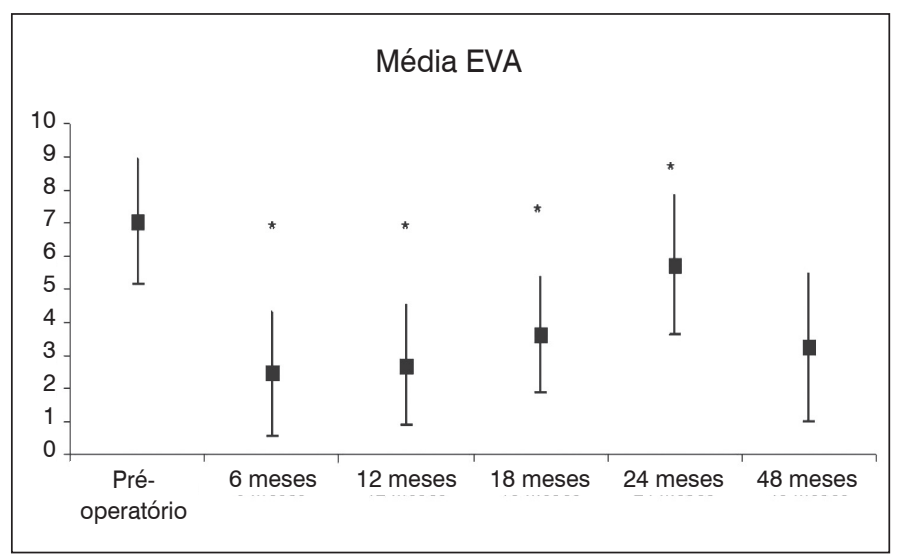

Figura 2. Valores médios do índice EVA ao longo do estudo, barra de erros representa o desvio padrão do grupo, * $p<0,05$.

\section{pós-operatório.}

Ocorreram duas complicações $(8,6 \%)$ menores - um paciente com parestesia transitória de L5 devido ao trauma mecânico do trocater durante o procedimento e outro com quadro de cefaléia persistente pós-punção de dura-máter.

\section{DISCUSSÃO}

A dor lombar com duração maior que seis meses refratária ao tratamento conservador é um desafio para o cirurgião de coluna ${ }^{24}$. O uso do IDET é bem aceito como uma opção terapêutica pouco invasiva. Os pacientes com LDI apresentam o dilema entre conviver com a dor, modificar o comportamento, depender de analgésicos ou submeter-se a cirurgia. A anuloplastia se encaixa numa zona cinzenta entre o tratamento conservador e o cirúrgico. O procedimento é realizado de forma percutânea, com a utilização de um cateter ablativo que não altera a anatomia e modifica pouco a estabilidade da coluna ${ }^{25}$.

A eficácia do IDET vem sendo estudada na literatura desde sua descrição inicial em 1997, por Saal e Saal ${ }^{17}$. Existe muita controvérsia sobre sua eficácia e os possíveis benefícios. A falta de comprovação dos efeitos biológicos pelos quais a IDET levaria ao alívio da dor gera descrédito por parte de alguns especialistas ${ }^{5}$. Os efeitos preconizados da eletrocoagulação das terminações nervosas nociceptivas no ânulo fibroso e da modificação da estrutura do colágeno estabilizando as fissuras anulares ainda não foram comprovados $^{2,17,25,26}$. Freeman et al. ${ }^{27}$ publicaram um trabalho em modelos ovinos, com a realização da anuloplastia em 16 animais e avaliação microscópica dos discos em 12 semanas após a data do procedimento. Foi verificado que não havia diminuição da quantidade de terminações nervosas nos discos submetidos ao procedimento quando comparado com os discos controles. Concluíram que os benefícios do IDET devem-se, aparentemente a outros fatores que não a denervação discal. Kleinstueck et al. ${ }^{26}$ mostraram que a temperatura necessária para produzir alterações na estrutura de colágeno $\left(60^{\circ}\right)$ é observada apenas no local do eletrodo (1 a $2 \mathrm{~mm}$ ). A teoria de que o IDET aumentaria a estabilidade do disco também foi descartada, verificando uma diminuição da rigidez de 6\% a 12\%.

Os trabalhos na literatura mostram resultados controversos, desde ausência completa de eficácia até resultados satisfatórios, duradouros com índices baixos de complicações $4,5,16,17,28-37$. A maioria desses trabalhos apresenta pouca força epidemiológica, geralmente são séries de casos de curto seguimento. Ainda hoje a literatura conta com um número pequeno de trabalhos sobre a evolução dos pacientes submetidos ao IDET num follow-up maior que dois anos. $\mathrm{O}$ estudo apresentado tem o follow-up com a médio de 32,55 meses, sendo que 35\% dos pacientes apresentam avaliação por mais de 48 meses.

Saal e $\mathrm{Saal}^{38}$ reportaram, em um estudo prospectivo com follow-up mínimo de dois anos, a melhora significativa e 
permanente da sintomatologia. Resultado concordante com os dados do estudo em questão, que mostrou evolução satisfatória na avaliação de 6, 12, 18 e 24 meses. Não houve diferença nos parâmetros clínicos estudados em relação ao pré-procedimento com o $48^{\circ}$ mês pós-operatório. A satisfação pessoal estava presente em $78 \%$ dos pacientes avaliados, concordante com Assietti e Morosi, que mostraram $78 \%$ para o IDET diante de pacientes bem selecionados: presença do HIZ ( higth intense zone), Pfirrmann menor ou igual a dois e a cobertura ampla do cateter no momento do procedimento ${ }^{39}$.

Ocorreram duas complicações (8,6\%) menores - os dois pacientes apresentaram melhora do quadro em poucos dias, não havendo sequelas. Russel et al..$^{33} \mathrm{em}$ uma analise prospectiva de 33 pacientes por 16 meses observou a quebra de um cateter e um prolapso discal com necessidade de discectomia após o procedimento. Apresentou 30\% de "pé caído" temporário, provavelmente devido aos agentes anestésicos. Não houve discite, infecção superficial ou profunda e lesão permanente de raízes. Já foi descrito a quebra do cateter, síndrome da cauda equina, osteonecrose do platô vertebral, bloqueio de nervos adjacentes e posicionamento errado do cateter produzindo lesões nervosas definitivas ${ }^{40,41}$.

O IDET foi eficaz para fornecer alivio temporário em até 24 meses, mas não mostrou-se capaz de alterar a história natural da lombalgia crônica. A partir da desse dado, fica o questionamento se o procedimento pode ser realizado no mesmo nível mais de uma vez. Não há trabalhos publicados sobre repetição do procedimento em pacientes que apresentaram recidiva.

\section{CONCLUSÃO}

O IDET mostrou resultados satisfatórios como alternativa paliativa no controle da dor e da incapacidade funcional num período de 24 meses. Os bons se deterioraram com 48 meses e houve uma tendência de retorno à sintomatologia inicial.

\section{REFERÊNCIA}

1. Cherkin DC, Deyo RA, Volinn E, Loeser JD. Use of the International Classification of Diseases (ICD-9-CM) to identify hospitalizations for mechanical low back problems in administrative databases. Spine (Phila Pa 1976). 1992;17(7):817-25.

2. McGraw JK, Silber JS. Intradiscal electrothemal therapy for the treatment of discogenic back pain. Aplied Radiology 2001: 30:11-16.

3. Lee J, Lutz GE, Campbell D, Rodeo SA, Wright T. Stability of the lumbar spine after intradiscal electrothermal therapy. Arch Phys Med Rehabil. 2001;82(1):120-2.

4. Biyani A, Andersson GB, Chaudhary H, An HS. Intradiscal electrothermal therapy: a treatment option in patients with internal disc disruption. Spine (Phila Pa 1976). 2003:28(15 Suppl):S8-14

5. Heary RF. Intradiscal electrothermal annuloplasty: the IDET procedure. J Spinal Disord. 2001;14(4):353-60.

6. Andersson $\mathrm{GB}$, Svensson $\mathrm{HO}$, Odén $\mathrm{A}$. The intensity of work recovery in low back pain Spine (Phila Pa 1976). 1983;8(8):880-4

7. Schwarzer AC, Aprill CN, Derby R, Fortin J, Kine G, Bogduk N. The prevalence and clinical features of internal disc disruption in patients with chronic low back pain. Spine (Phila Pa 1976). 1995;20(17):1878-83.

8. O'Neill C, Derby R, Kenderes L. Precision Injection Techniques for diagnosis and treatment of lumbar disc disease. Sem. Spine Surg. 1999: 11: 104-18.

9. Boden SD, Davis DO, Dina TS. The incidence of abnormal lumbar spine: MRI scans in asyntomatic patients: A prospective and blinded investigation. Orthop Trans. 1990;14:66.

10. Colhoun E, McCall IW, Williams L, Cassar Pullicino VN. Provocation discography as a guide o planning operations on the spine. J Bone Joint Surg Br. 1988;70(2):267-71.

11. Jackson RP, Becker GJ, Jacobs RR, Montesano PX, Cooper BR, McManus GE. The neuroradiographic diagnosis of lumbar herniated nucleus pulposus: I. A comparison of computed tomography (CT), myelography, CT-myelography, discography, and CT-discography. Spine (Phila Pa 1976). 1989;14(12):1356-61. Erratum in: Spine. 1990;15(1):59.

12. Kornberg M. Discography and magnetic resonance imaging in the diagnosis of lumbar disc disruption. Spine (Phila Pa 1976). 1989:14(12):1368-72.

13. Schwarzer AC, Aprill CN, Derby R, Fortin J, Kine G, Bogduk N. The relative contributions of the disc and zygapophyseal joint in chronic low back pain. Spine (Phila Pa 1976). 1994;19(7):801-6.

14. Crock HV. A reappraisal of intervertebral disc lesions. Med J Aust. 1970;1(20):983-9

15. Crock HV. Internal disc disruption. A challenge to disc prolapse fifty years on. Spine (Phila Pa 1976). 1986;11(6):650-3.

16. Wetzel FT, McNally TA, Phillips FM. Intradiscal electrothermal therapy used to manage chronic discogenic low back pain: new directions and interventions. Spine (Phila Pa 1976). 2002;27(22):2621-6

17. Saal JA, Saal JS. For the treatment of chronic discogenic low back pain. Operative Techn Orthop. 2000;10:271-80.

18. Wetzel FT, LaRocca SH, Lowery GL, Aprill CN. The treatment of lumbar spinal pain syndromes diagnosed by discography. Lumbar arthrodesis. Spine (Phila Pa 1976). 1994;19(7):792-800.

19. Wetzel FT, McNally TA. Treatment of chronic discogenic low back pain with intradiskal electrothermal therapy. J Am Acad Orthop Surg. 2003;11(1):6-11.

20. Chou R, Baisden J, Carragee EJ, Resnick DK, Shaffer WO, Loeser JD. Surgery for low back pain: a review of the evidence for an American Pain Society Clinical Practice Guideline. Spine (Phila Pa 1976). 2009;34(10):1094-109.

21. Horton WC, DaftariTK. Which disc as visualized by magnetic resonance imaging is actually a source of pain? A correlation between magnetic resonance imaging and discography. Spine (Phila Pa 1976). 1992;17(6 Suppl):S164-71.

22. Fairbank JC, Couper J, Davies JB, O'Brien JP. The Oswestry low back pain disability questionnaire. Physiotherapy. 1980;66(8):271-3.
23. Walsh TR, Weinstein JN, Spratt KF, Lehmann TR, Aprill C, Sayre H. Lumbar discography in normal subjects. A controlled, prospective study. J Bone Joint Surg Am. 1990;72(7):1081-8.

24. Von Korff M. Studying the natural history of back pain. Spine (Phila Pa 1976). 1994 Sep;19(18 Suppl):2041S-2046S.

25. Kleinstueck FS, Diederich CJ, Nau WH, Puttlitz CM, Smith JA, Bradford DS, et al. Temperature and thermal dose distributions during intradiscal electrothermal therapy in the cadaveric lumbar spine. Spine (Phila Pa 1976). 2003;28(15):1700-8.

26. Kleinstueck FS, Diederich CJ, Nau WH, Puttlitz CM, Smith JA, Bradford DS, et al. Acute biomechanical and histological effects of intradiscal electrothermal therapy on human lumbar discs. Spine (Phila Pa 1976). 2001;26(20):2198-207.

27. Freeman BJC, RM Walters, RJ Moore, B Vernon-Roberts, RD Fraser. AN Assessment Of The Potential For Intra-Discal Lectrothermal Therapy (Idet) To Denervate Posterior Annular Lesions. J Bone Joint Surg Br. 2003;85:(Suppl 2):165.

28. Pauza KJ, Howell S, Dreyfuss P, Peloza JH, Dawson K, Bogduk N. A randomized, placebo-controlled trial of intradiscal electrothermal therapy for the treatment of discogenic low back pain. Spine J. 2004;4(1):27-35.

29. Davis TT, Delamarter RB, Sra P, Goldstein TB. The IDET procedure for chronic discogenic low back pain. Spine (Phila Pa 1976). 2004;29(7):752-6.

30. Bogduk N, Karasek M. Two-year follow-up of a controlled trial of intradiscal electrothermal anuloplasty for chronic low back pain resulting from internal disc disruption. Spine J. 2002:2(5):343-50.

31. Jones HW, McClean C, Marsh G. Preoperative pain drawings as predictors of success of intradiscal electrothermal therapy in the treatment of chronic discogenic low back pain. J. Bone Joint Surg. 2003; 85B (Suppl III): 237

32. Russell RC, Corbett $\mathrm{S}$, March G. An early european experience with IDET - Average follow-up of 20 months. J. Bone Joint Surg. [Br] 2002; 85B (Suppl II): 170

33. Ahuja S, Russell ID, Howes J, Davis PR. Intra-discal electrothermal therapy: a minimum of 18 months prospective follow-up study. J. Bone Joint Surg. 2003; 85B (Suppl II): 164.

34. Bogduk N, Aprill C, Derby R. Discography. In: White AH, Arthur H. Spine Care vol.1 Diagnosis and conservative treatment. St Louis: Mosby; 1995. p 219-238.

35. Freeman BJ, Fraser RD, Cain C, Hall DJ. A Randomized double-blind controlled efficacy study, intradiscal electrothermal therapy (IDET) versus placebo. J. Bone Joint Surg.2004; 86B (Suppl IV): 484-85

36. Freedman BA, Cohen SP, KukloTR, Lehman RA, Larkin P, Giuliani JR. Intradiscal electrothermal therapy (IDET) for chronic low back pain in active-duty soldiers: 2-year follow-up. Spine J. 2003;3(6):502-9.

37. Freeman BJ, Fraser RD, Cain CM, Hall DJ, Chapple DC. A randomized, double-blind, controlled trial: intradiscal electrothermal therapy versus placebo for the treatment of chronic discogenic low back pain. Spine (Phila Pa 1976).2005;30(21):2369-77.

38. Saal JA, Saal JS. Intradiscal electrothermal treatment for chronic discogenic low back pain: prospective outcome study with a minimum 2-year follow-up. Spine (Phila Pa 1976). 2002;27(9):966-73.

39. Assietti R, Morosi M, Block JE. Intradiscal electrothermal therapy for symptomatic internal disc disruption: 24-month results and predictors of clinical success. J Neurosurg Spine. 2010;12(3):320-6.

40. Nachemson A. Lumbar discography--where are we today? Spine (Phila Pa 1976). 1989;14(6):555-7.

41. Bhagia SM, Slipman CW, NirschI M, Isaac Z, El-Abd O, Sharps LS, et al. Side effects and complications after percutaneous disc decompression using coblation technology. Am J Phys Med Rehabil. 200; 85(1):6-13. 